\title{
Mobilização precoce no doente crítico: atualização
}

O trabalho tem como objetivo analisar e identificar os efeitos e as repercussões dos protocolos de mobilização precoce publicados, correlacionando os resultados obtidos com as variáveis analisadas em cada estudo, com a evolução clínica do doente crítico. Os materiais e métodos utilizados foram a revisão bibliográfica integrativa de caráter narrativo, como o objetivo de identificar os protocolos de mobilização precoce disponibilizados. Abrangendo publicações disponibilizados na base de dados em sites acadêmicos oficiais e confiáveis, revistas científicas especializadas em fisioterapia e terapia intensiva, base de dados do programa de pósgraduação de algumas universidades e livros atualizados dos últimos 10 anos. Apolineuromiopatia do doente crítico é complexa e multifatorial, tendo como característica: perda de força generalizada, perda de massa muscular, podendo ser acompanhada por alteração de sensibilidade. Tem acometimento predominante motor, de natureza axonal, simétrico e agudo, os pacientes que desenvolvem apresentam dificuldade no desmame da ventilação mecânica, além da tetraparesia e arreflexia. A intitulação de protocolos baseados em evidências científicas na UTI, auxilia na otimização e padronização do atendimento. Sendo hoje inquestionável seu uso por seu resultado ser melhora no desfecho clínico. Sugere-se a realização de ensaios clínicos com maior padronização para descrição e comparação de diferentes protocolos de tratamento, com objetivo de identificar e determinar a dose ideal, o tempo de exercício e o efeito do exercício em condições específicas. Embora a eficácia das intervenções de fisioterapia não seja para debate, os mecanismos fisiopatológicos de intervenções específicas e a relação dose-resposta em pacientes com terapia intensiva permanecem desconhecidos.

Palavras-chave: Mobilização Precoce; Fisioterapia; Cinesioterapia; Protocolo; Paciente Crítico.

\section{Early mobilization in the critical patient: update}

The objective of this study was to analyze and identify the effects and repercussions of published early mobilization protocols, correlating the results obtained with the variables analyzed in each study, with the clinical evolution of the critical patient. The materials and methods used were the integrative bibliographical revision of narrative character, as the objective of identifying the protocols of early mobilization made available. Covering publications made available in the database on official and reliable academic sites, scientific journals specialized in physiotherapy and intensive therapy, database of the graduate program of some universities and updated books of the last 10 years. Apolineuromiopathy of the critical patient is complex and multifactorial, having as characteristic: loss of generalized force, loss of muscular mass, being able to be accompanied by alteration of sensitivity. It has predominant motor involvement, axonal, symmetrical and acute, the patients who develop have difficulty in weaning mechanical ventilation, in addition to tetraparesis and arreflexia. The titling of protocols based on scientific evidence in the ICU, helps in the optimization and standardization of care. Today its use is unquestionable because its result is improvement in the clinical outcome. It is suggested to carry out more standardized clinical trials for the description and comparison of different treatment protocols, in order to identify and determine the ideal dose, the time of exercise and the effect of the exercise under specific conditions. Although the effectiveness of physiotherapy interventions is not for debate, the pathophysiological mechanisms of specific interventions and the dose-response relationship in intensive care patients remain unknown.

Keywords: Early Mobilization; Physiotherapy; Kinesiotherapy; Protocol; Critical Patient.

Topic: Fisioterapia

Reviewed anonymously in the process of blind peer.
Received: $\mathbf{1 3 / 1 1 / 2 0 1 7}$

Approved: 23/01/2018

Danilo Cavalcante Gonçalves

Instituto de Excelência em Educação e Saúde, Brasil.

http://lattes.cnpq.br/4076738155896803

danilocavalcante2006@ hotmail.com

Deyse Lorenna Batista Martins

danilocavalcante2006@hotmail.com

Referencing this:

GONÇALVES, D. C.; MARTINS, D. L. B.. Mobilização precoce no doente crítico: atualização. Scire Salutis, v.8, n.1, p.16-24, 2018. DOI: http://doi.org/10.6008/CBPC2236-9600.2018.001.0003 


\section{INTRODUÇÃO}

À medida que a sobrevida dos pacientes criticamente enfermos aumenta, em consequência dos avanços tecnológicos e do olhar cada vez mais multidisciplinar dos intensivistas, cresce também o interesse pelas repercussões causadas pelo imobilismo durante os períodos de permanência em internação como também em ventilação mecânica. Os pacientes que sobrevivem a esses agravos, sobretudo quando associado à internação prolongada, apresentam graves problemas psicológicos e físico após a alta hospitalar, afetando à qualidade de vida e aumentando a mortalidade (SANDRI et al., 2014, citado por PEREIRA, 2014; FRANÇA et al., 2012; FRANÇA et al., 2010).

Na maioria das vezes os pacientes críticos admitidos em uma unidade de terapia intensiva (UTI) requerem longos períodos de internação, estando submetidos ao imobilismo, que resulta em perda significativa de massa muscular e força, com cerca de 1 a $5 \%$ por dia em repouso no leito, além de serem acometidos por efeitos adversos sistêmicos e complicações, tais como: úlcera de decúbito, atrofia muscular, delirium, atelectasias, pneumonia, hipotensão ortostática e trombose venosa profunda (SANDRI et al., 2014, citado por PEREIRA, 2014).

Durante um longo período, esse padrão de cuidado, foi mantido dentro das unidades, porém, com novos estudos na última década foi observado os efeitos benéficos da mobilização precoce nas complicações próprias da imobilidade, como também seu efeito fisiológico de modo preventivo. Nesse sentido, a literatura atual traz uma nova tendência no manejo do paciente crítico, incluindo redução de sedação profunda, mobilização precoce e manutenção da capacidade funcional. Mostrando claramente que pacientes submetidos a um protocolo de mobilização precoce apresentam menor tempo de internação e até redução da mortalidade, além de reduzir os índices de delírio (SANDRI et al., 2014, citado por PEREIRA, 2014; FRANÇA et al., 2010). Por essa razão, este artigo teve por objetivo analisar e Identificar os efeitos e as repercussões dos protocolos de mobilização precoce publicados, correlacionando os resultados obtidos com as variáveis analisadas em cada estudo, com a evolução clínica do doente crítico.

\section{METODOLOGIA}

O presente estudo trata-se de uma revisão bibliográfica integrativa de caráter narrativo, como o objetivo de identificar os protocolos de mobilização precoce disponibilizados. Abrangendo publicações de disponibilizados na base de dados SCIELO, Google Acadêmico, revistas científicas especializadas em fisioterapia e terapia intensiva, base de dados do programa de pós-graduação da Universidade Federal de Minas Gerais/UFMG e Universidade Federal do Rio Grande do Sul/UFRGS.

Os critérios de inclusão adotados na pesquisa foram: publicações compreendidas no período de 2007 a 2017, que fosse possível entender a aplicabilidade do protocolo de mobilização precoce proposto com resultados obtidos. Foram selecionados livros de atualização de conduta terapeuta para fisioterapeutas, força tarefa e recomendações da Associação de medicina Intensiva (AMIB), Associação Brasileira de Fisioterapia em Terapia Intensiva (Assobrafir) e European Respiratory Society and Europen Societyof 
Intensive Care Medicine. Estudos clínicos com disponibilidade de acesso na integra, por essa razão foram excluídos da pesquisa, artigos com falta de delimitação da metodologia de pesquisa, estudos anteriores à média proposta dos últimos 10 anos.

\section{DISCUSSÃO TEÓRICA}

\section{Fraqueza adquirida na UTI}

No período de internação, com imobilismo, os pacientes passam por uma transformação de fibras musculares para o tipo II, incluindo a redução da capacidade oxidativa, da densidade mitocondrial e de capilares sanguíneos. A performance cardiovascular encontra-se reduzida, devido ao menor volume de ejeção sistólico e ao aumento da frequência cardíaca. A estase venosa, devido à redução da atividade da bomba muscular nos membros, aumenta o risco do desenvolvimento de trombose, podendo também, contribuir para a desmineralização óssea e a redução do sódio e água corporal (FRANÇA et al., 2010).

São várias as condições clínicas que predispõe os pacientes críticos a fraqueza muscular. Descrita inicialmente em pacientes asmáticos que faziam uso de corticoesteroides e bloqueadores muscular e mais frequentemente em pacientes que sofre de insuficiência respiratória aguda, síndrome da resposta inflamatória aguda (SIRS) e sepse. Sendo de fundamental importância para melhor evolução à avaliação para diagnóstico funcional prévio com a escala da Medida de Independência Funcional (MIF), e assim quantificar a perda funcional durante internamento (FRANÇA et al., 2012).

Denominada polineuromiopatia do doente crítico, tal acometimento é complexo e multifatorial, tendo como característica: perda de força generalizada, perda de massa muscular, podendo ser acompanhada por alteração de sensibilidade. Tem acometimento predominante motor, de natureza axonal, simétrico e agudo, os pacientes que desenvolvem apresentam dificuldade no desmame da ventilação mecânica, além da tetraparesia e arreflexia (BUTTIGNOL et al., 2016, citado por ASSOBRAFIR, 2016; SANDRI et al., 2014, citado por PEREIRA, 2014).

A avaliação detalhada para detecção precoce de qualquer sinal de fraqueza muscular e a atuação da equipe multidisciplinar, com o objetivo de diminuir a exposição aos fatores de risco que podem desencadear a polineuromiopatia do doente crítico são de grande importância. Os principais critérios para o diagnóstico são: fraqueza associada à doença crítica; fraqueza bilateral, de característica flácida, envolvendo musculatura distal e proximal, geralmente não acomete nervos cranianos; pontuação geral pela estala de MRC (Medical Research Council), como pode ser visto na Tabela 1; ventilação mecânica prolongada; e exclusão de outras causas de fraqueza muscular.

O diagnóstico fisioterapêutico, também definido como diagnóstico cinesiofuncional, não é estático e é pré-requisito para a conduta fisioterapêutica, impondo ao profissional uma avaliação continuada no processo de conduta de um caso clínico, reforçando a noção de que o foco terapêutico não é para doença em si, e sim para os eventos semiológicos oriundos dessa situação. Nesse contexto o efetivo é avaliar os 
resultados para que se possa testar a validade dos processos diagnósticos e terapêutico, a fim de conduzi-los com maior propriedade científica (FRANÇA et al., 2012).

Tabela 1: Escala modificada de MRC (Medical Research Council) para avaliação de força muscular.

\begin{tabular}{|l|l|}
\hline $\mathbf{0}$ & Ausência de contração muscular. \\
\hline $\mathbf{1}$ & Presença de contração muscular, sem amplitude de movimento. \\
\hline $\mathbf{2}$ & Apresenta movimento ativo, não completa amplitude de movimento. \\
\hline $\mathbf{3}$ & Apresenta movimento ativo, completa amplitude de movimento. \\
\hline $\mathbf{4}$ & Apresenta movimento ativo contra a gravidade resistência. \\
\hline $\mathbf{5}$ & Força muscular normal. \\
\hline
\end{tabular}

Fonte: Buttignol et al. (2014, citado por ASSOBRAFIR, 2014); Sandri et al. (2014, citado por PEREIRA, 2014).

\section{Mobilização precoce}

A mobilização precoce vem se tornando uma realidade presente nas unidades de cuidados intensivos, liderando as condutas fisioterapêutica. Realidade essa que vem sendo construída dentro da equipe multidisciplinar, pois, a implementação de um programa de reabilitação com mobilização precoce, depende de uma série de recursos, tempo e sobretudo cooperação de toda equipe (SANDRI et al., 2014, citado por PEREIRA, 2014).

A estruturação de um programa de mobilização precoce requer do fisioterapeuta uma avaliação continuada do estado de saúde do indivíduo. Devendo levar em consideração os seguintes tópicos: Análise da história médica e medicamentos; nível funcional antes da internação; nível de consciência; condição neurológica; estabilidade hemodinâmica; estabilidade respiratória; condição musculoesquelética; integridade da pele; avaliação funcional, objetivos e plano de tratamento (ALBUQUERQUE et al., 2015; AZEVEDO et al., 2015; SANDRI et al., 2014, citado por PEREIRA, 2014).

A força-tarefa da European Respiratory Society and Europen Societyof Intensive Care Medicine estabeleceu uma hierarquia de atividades de mobilização na UTI com base em uma sequência de intensidade de exercícios, partindo da mudança de decúbito, posicionamento funcional e progresso. Com grau de recomendação Nível B, para mobilização precoce e Ortostatismo. Recomenda ainda que o Fisioterapeuta seja o profissional responsável pela implantação e pelo gerenciamento do plano de mobilização, o qual reflete diretamente para o treinamento de futura atividades funcionais (FRANÇA et al., 2012; FRANÇA et al., 2010; GOSSELINK et al., 2008).

O departamento de Fisioterapia da Associação de Medicina intensiva brasileira, publicou em 2012 recomendações mínimas aplicáveis à realidade brasileira, com critérios e mecanismos de segurança na mobilização precoce do doente crítico (FRANÇA et al., 2012). As atividades propostas por meio de protocolos, os quais demonstraram ser seguras e viáveis por alguns estudos, devendo ser iniciada o mais precoce possível, após a estabilização de quadros de choque não controlados. A monitorização durante e após o exercício é mandatória, recomenda-se a avaliação das variáveis cardiovasculares (frequência cardíaca e pressão arterial) e respiratórias (padrão muscular ventilatório do paciente e sincronia do paciente com o ventilador mecânico, saturação periférica de oxigênio e frequência respiratória) (FRANÇA et al., 2012).

A avaliação da dosagem de sedativos, drogas vasoativas e oferta de oxigênio, pois, pacientes com instabilidade hemodinâmica com necessidade de altas frações inspiradas de oxigênio (FiO2) e altos níveis de 
suporte ventilatório, não são recomendados para atividades de mobilização de alta intensidade (FRANÇA et al., 2012). O status fisiológico do paciente crítico pode ser alterado com facilidade ao longo do dia, o que pode dificultar a realização dos exercícios físicos, sendo assim necessário a elaboração de um planejamento individualizado e com maior flexibilidade possível, baseando-se na avaliação beira leito continuada dos candidatos à mobilização como descrito na Figura 1 (FRANÇA et al., 2012).

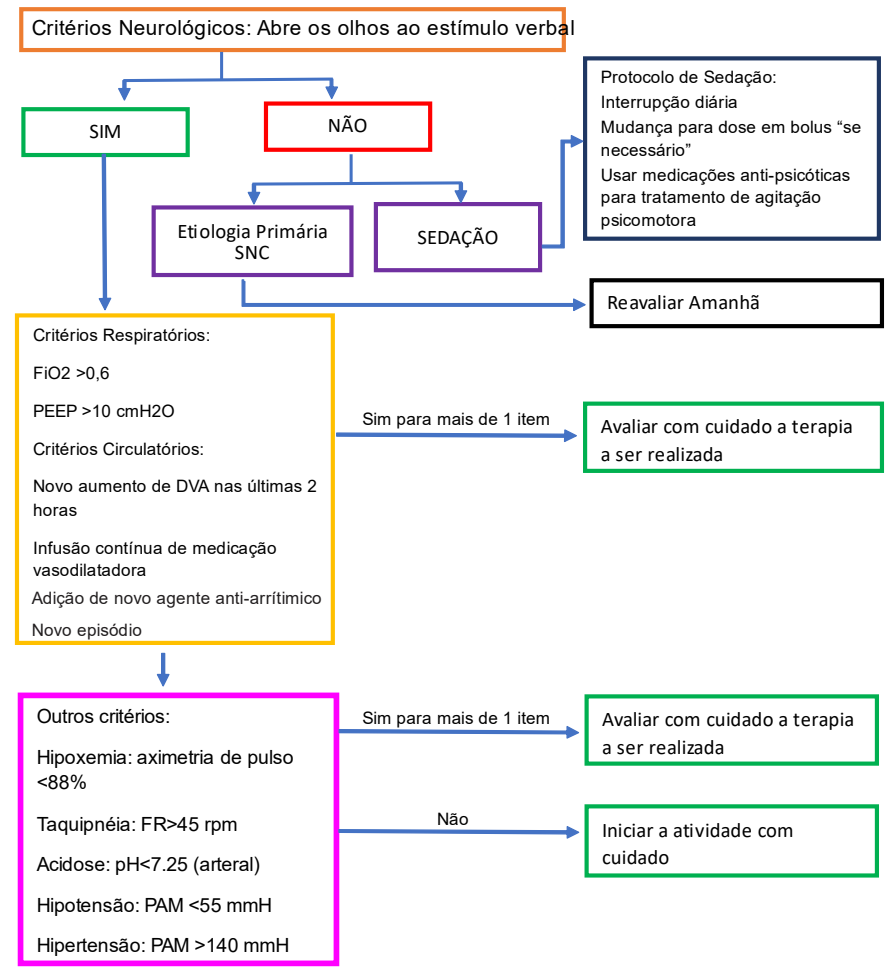

Figura 1: Algoritmo de avaliação dos pacientes candidatos ao treinamento em pacientes críticos. Fonte: FRANÇA et al., 2012. Legenda: SNC: sistema nervoso central; PAM: pressão arterial média; FiO2: fração inspirada de oxigênio; PEEP: positive expiratoryendpressure; DVA: droga vasoativa; FR: frequência respiratória; TVP: trombose venosa profunda.

É unanime os benefícios da mobilização precoce comparando a terapia padrão, quando não inclui exercícios ativos e retirada do leito, assim, dentre esses benefícios, podemos citar: diminuição do tempo de ventilação mecânica; diminuição do tempo de internação na UTI e hospitalar; aumento da força muscular; manutenção/recuperação do grau prévio de funcionalidade dos pacientes internados; diminuição do número de quadros infecciosos; diminuição do delírio e; por resultado de todo processo melhora na qualidade de vida (BUTTIGNOL et al., 2016, citado por ASSOBRAFIR, 2016).

\section{Protocolos de Mobilização precoce}

A intitulação de protocolos baseados em evidências científicas na UTI, auxilia na otimização e padronização do atendimento. Sendo hoje inquestionável seu uso por seu resultado ser melhora no desfecho clínico. Não há uma padronização nos estudos encontrados com variações que não nos deixam fazer uma correlação exata de respostas aos exercícios devido a especificações das populações estudadas, tipos de unidades, estruturas disponíveis, intensidade, dose, tempo e tipos de exercícios aplicados (BUTTIGNOL et al., 2016, citado por ASSOBRAFIR, 2016) 
Um ensaio clínico aleatorizado concluiu que a mobilização precoce é segura, eficaz e favorece a saída precoce do leito, principalmente quando iniciada nas primeiras 24 horas de internação, sendo clinicamente relevante em todos os contextos, reduzindo o tempo de internação na UTI e os custos totais. O Protocolo de Gosselink et al. (2012), foi descrito nas recomendações brasileiras de fisioterapia no paciente crítico adulto, aplicado em cinco fases, como dispunha na Tabela 2.

Tabela 2: Recomendações Brasileiras de Fisioterapia.

\begin{tabular}{|c|l|}
\hline Fase $\mathbf{0}$ & Instável com restrição médica de mobilização. Mudança de decúbito a cada 3 horas (orientar enfermagem); \\
\hline Fase $\mathbf{1}$ & $\begin{array}{l}\text { Baixo nível de cooperação impossibilidade de permanecer sentado. Mudança de decúbito a cada } 3 \text { horas, cabeceira } \\
\text { elevada, mobilização passiva manual; }\end{array}$ \\
\hline Fase $\mathbf{2}$ & $\begin{array}{l}\text { Nível de compreensão moderado. MRC }>36 \text {. Com contraindicação médica para sair do leito ativamente. Mudança de } \\
\text { decúbito a cada } 3 \text { horas, sedestação no leito, transferência passiva para cadeira. Mobilização ativo/assistida, } \\
\text { ativo/resistida MMII MMSS; }\end{array}$ \\
\hline Fase $\mathbf{3}$ & $\begin{array}{l}\text { Nível de compreensão quase completo. MRC }>36 \text {. Exercícios ativo/assistida, treino de ADV's, transferência ativo } \\
\text { assistida, exercícios de resistência MMSS MMII, sedestação no leito sem apoio posterior. Ortostatismo com } 2 \text { apoios; }\end{array}$ \\
\hline Fase $\mathbf{4}$ & $\begin{array}{l}\text { Nível de compreensão alto, MRC }>48, \text { transferência ativa para cadeira, sentar no leito sem apoio, Ortostatismo com } \\
\text { apoio de uma pessoa, treino de ADV's, exercícios ativo/assistido, resistidos e treino de marcha estacionária; }\end{array}$ \\
\hline Fase $\mathbf{5}$ & $\begin{array}{l}\text { Alto nível de compreensão, MRC }>48, \text { sentado para Ortostatismo com apoio mínimo e se estabilizar, treino de ADV's, } \\
\text { deambula com apoio mínimo ou nenhum, exercício ativos assistidos e resistidos. }\end{array}$ \\
\hline
\end{tabular}
Fonte: GOSSELINK et al. (2012).

Buttignol et al. (2016, citado por ASSOBRAFIR, 2016), em uma revisão, descreveram os protocolos clínicos mais utilizados nos estudos publicados em revistas, podendo assim servir de guia para a elaboração de uma ferramenta que se adeque a realidade local. Morris et al. (2008) teve 330 pacientes em seu estudo, divididos em Grupo controle (atendimento prestado quando solicitado) e Grupo intervenção (mobilização precoce 3 vezes ao dia seguindo escala de evolução). Foram incluídos pacientes com insuficiência respiratória em VM >72 horas de admissão. Os pacientes alocados no grupo de intervenção apresentaram menor tempo para sair da cama, menor tempo de hospitalização na UTI e hospitalar

Schweickert et al. (2009) teve 104 pacientes, divididos em Grupo controle - não receberam fisioterapia; Grupo intervenção - mobilização precoce. Foram selecionados pacientes adultos sob VM >72 horas na UTI, que apresentassem independência funcional antes da internação. O retorno ao estado funcional prévio, menor tempo de VM e delírio foram melhores no grupo intervenção. Em três revisões sistematizadas publicadas no Brasil com descrição dos estudos clínicos publicados foi possível correlacionar e identificar as diferenças e resultados obtidos nos mesmos (ALBUQUERQUE et al., 2015; AZEVEDO et al., 2015; SILVA et al., 2014).

Silva et al. (2014) analisou 875 estudos, sendo selecionados oito ensaios clínicos para investigação, destes, em UTIs europeias, norte americanas, asiática e brasileira, sendo observado melhora da força muscular, aumento da independência funcional, menor tempo de ventilação mecânica, baixo nível de eventos adversos após protocolo de exercícios sem aumento de custos. Já Azevedo et al. (2015) em sua revisão relata que as limitações encontradas em sua análise decorrem das características intrínsecas da complexidade das respostas do doente crítico à doença, como também a ausência de um instrumento consistente que correlacione a melhora dos níveis de forças com a mobilização precoce na fraqueza muscular resultante da administração medicamentosa. 
Albuquerque et al. (2015) concluiu que há evidências de que programas de mobilização precoce são seguros, melhoram o desempenho funcional na alta da UTI, reduzem a incidência de delirium, bem como reduzem o tempo de ventilação mecânica e permanência hospitalar. Ressaltando que os níveis de evidência da mobilização precoce sobre desfechos clínicos relevantes, como tempo de permanência em UTI, mortalidade hospitalar e a sobrevida a longo prazo são baixos com necessidade de estudos.

Em um consenso de especialistas com recomendações sobre os critérios de segurança para mobilização precoce ativa em pacientes críticos em ventilação mecânica adulto, foi subdividido em quatro categorias: respiratória, cardiovascular, neurológica e outras. Sendo identificado com cores do semáforo de baixo risco adverso (verde), risco potencial de um evento adverso compensado pelo benefício da mobilização (amarelo) e risco potencial significativo de um evento adverso (vermelho). Estar sob ventilação mecânica para mobilização precoce ativa, uma fração inspirada de oxigênio menor que $60 \%$ com uma saturação de oxigênio superior a 90\%, com frequência respiratória inferior à 30 respirações por minuto foram consideradas critérios para retirar do leito quando não associado à outras contraindicações (HODGSON et al., 2014).

O estudo AVERT com objetivo de fornecer orientações quanto a dose-resposta na mobilização precoce do paciente portador de acidente vascular cerebral (AVC), demostrou a eficácia da atuação nos pacientes com início nas primeiras 24 horas dos sintomas clínicos acompanhados até o terceiro mês de evolução. Sugerindo melhores resultados quando intitulada uma mobilização de curta duração, maior frequência no início está associada a maior probabilidade de desfecho favorável para recuperação da independência pós AVC (BERNHARDT et al., 2016).

No estudo de Dafoe et al. (2015) onde analisou as barreiras encontradas uma UTI na Austrália, identificou que os fatores relacionados ao paciente e à instituição tiveram maior impacto na mobilização dos pacientes. As barreiras identificadas como passíveis de mudanças incluíram educação insuficiente sobre os benefícios da mobilização, comunicação interdisciplinar e falta de liderança em relação a mobilização. Com implantação de treinamento e conscientização da equipe multidisciplinar por 3 meses, não fui visualizado mudança no padrão da mobilização, sugerindo, assim, mudança na prática de sedação para assim possibilitar evolução de etapas.

É cada dia mais claro a importância de uma equipe treinada com foco na melhora clínica do paciente para tomada de decisão, buscando facilitar o processo com o objetivo de promover qualidade no atendimento multidisciplinar. Sendo fundamental a implementação na unidade de um fluxograma descrevendo a avaliação clínica do paciente correlacionando as responsabilidades da equipe durante o processo, incluindo o fisioterapeuta, médico, enfermeiro e técnico de enfermagem no processo de retirada do leito, assegurando, com isso, ao paciente, segurança e melhor qualidade no procedimento (GREEN et al., 2015). 


\section{CONCLUSÕES}

As evidências científicas para os benefícios da mobilização precoce em pacientes criticamente enfermos são cada vez mais presentes na literatura nacional e internacional. Neste artigo, foram apresentados os principais protocolos de atendimento fisioterapêutico utilizados nos estudos até o momento, com as principais barreiras para a mobilização precoce e os critérios de segurança, iniciando um conceito até então pouco comentado, que é a avaliação de fragilidade em pacientes graves internados em UTI. O uso consciente destas informações (avaliação do paciente e aplicação dos protocolos de intervenção) auxilia na prescrição do exercício e levará à otimização do atendimento ao paciente, bem como a melhores desfechos de saúde e melhor avaliação dos resultados do tratamento.

Em virtude da falta de evidências de superioridade de um protocolo em relação ao outro, sugere-se que os profissionais discutam com suas equipes e adaptem o melhor protocolo para sua população e seu serviço. Sugere-se a realização de ensaios clínicos com maior padronização para descrição e comparação de diferentes protocolos de tratamento, com objetivo de identificar e determinar a dose ideal e o tempo de exercício, bem como o efeito do exercício em condições específicas. Embora a eficácia das intervenções de fisioterapia não seja para debate, os mecanismos fisiopatológicos de intervenções específicas e a relação dose-resposta em pacientes com terapia intensiva permanecem desconhecidos.

\section{REFERÊNCIAS}

ALBUQUERQUE, I. M. CARVALHO, M. T. X.; SOARES, J. C.; MACHADO, A. S.. Impacto da mobilização precoce em pacientes de terapia intensiva. Salud(i) Ciência, v.21, n.4, p.403-408, 2015.

AZEVEDO, P. M. D. S.; GOMES, B. P.. Efeitos da mobilização precoce na reabilitação funcional em doentes críticos: uma revisão sistemática. Revista de Enfermagem Referência, v.5, n.4, p.129-138, 2015.

BERNHARDT, J.; CHURILOV, L.; ELLERY, F.; COLLIER, J.; CHAMBERLAIN, J.; LANGHORNE, P.; LINDLEY, R. I.; MOODIE, M.; DEWEY, H.; THRIFT, A. G; DONNAN, G.. Prespecified dose-response analysis for a very early rehabilitation trial (AVERT). American Academy of Neurology, v.86, n.7, p.2138-2145, 2016.

BUTTIGNOL M.; PIRES, N. R. C.. Protocolos de mobilização precoce no paciente crítico. In: ASSOBRAFIR; DIAS C.M.; MARTINS J. A.. Associação Brasileira de Fisioterapia em Terapia Intensiva. PROFISIO: Terapia Intensiva Adulto. Porto Alegre: Artmed Panamericana, 2014.

BUTTIGNOL, M.; PIRES, N. R. C.; ANNONI, R.. Protocolos de mobilização precoce no paciente crítico: up-to-date. In: ASSOBRAFIR; MARTINS J. A.; ANDRADE, F. M. D.; BERALDO, M. A.. Associação Brasileira de Fisioterapia em Terapia Intensiva. PROFISIO, Terapia Intensiva Adulto. Porto Alegre: Artmed Panamericana, 2016.

DAFOE, S.; CHAPMAN, M. J.; EDWARDS, S.. Overcoming barriers to the mobilization of patients in an intensive care unit. Austrália. AnaesthIntensive Care, v.43, n.6, p.719-727, 2015.
FRANÇA, E. E. T. FERRARI, F.; FERNANDES, P.; CAVALCANTI, R.; DUARTE, A.; MARTINEZ, B. P.; AQUIM, E. E.; DAMASCENO, M. C. P.. Fisioterapia em pacientes críticos adulto: recomendações do departamento de fisioterapia da Associação de Medicina Intensiva Brasileira. Revista Bras. Ter. Intensiva, v.24, n.1, p.6-22, 2012.

FRANÇA, E. E. T. FERRARI, F.; FERNANDES, P.; CAVALCANTI, R.; DUARTE, A.; MARTINEZ, B. P.; AQUIM, E. E.;

DAMASCENO, M. C. P.. Mobilização no paciente crítico: procedimentos e evidências. In: ASSOBRAFIR; GUIMARÃES, F. S.. Associação Brasileira de Fisioterapia em Terapia Intensiva. PROFISIO: Terapia Intensiva adulto. Porto Alegre, Porto Alegre. 2010.

GOSSELINK, R.; BOTT, J.; JOHNSON, M.; DEAN, E.; NAVA, S.; NORRENBERG, M.; SCHÖNHOFER, B.; STILLER, K.; VAN DE LEUR, H.; VINCENT, J. L.. Physiotherapy for adult patients with critical illness: recommendations of the european respiratory society and europeansociely of intensive care medicine task force on physiotherapy for critically ill patients. Intensive Care Med., v.34, n.7, p.1188-1199, 2008.

GREEN, $M$ et al. Mobilization of intensive care patients: a multidisciplinary practical guide for clinicians. Australia. Journal of Multidisciplinary Healthcare. 25(9), 2016: p. 247256.

HODGSON, C. L.; STILLER, K.; NEEDHAM, D. M.; TIPPING, C. J.; HARROLD, M.; BALDWIN, C. E.; BRADLEY, S.; BERNEY, S.; CARUANA, L. R.; ELLIOTT, D.;GREEN, M.; HAINES, K.; HIGGINS, A. M.; KAUKONEN, K. M.; LEDITSCHKE, I. A.; NICKELS, M. R.; PARATZ, J.; PATMAN, S.; SKINNER, E. H.; 
YOUNG, P. J.; ZANNI, J. M.; DENEHY, L.; WEBB, S. A.. Expert consensus and recommendations on safety criteria for active mobilization of mechanically ventilated critically ill adults. Critical Care, v.18, n.6, p.1-9, 2014.

MORRIS, P. E.; GOAD, A.; THOMPSON, C.; TAYLOR, K.; HARRY, B.; PASSMORE, L.; ROSS, A.; ANDERSON, L.; BAKER, S.; SANCHEZ M.; PENLEY, L.; HOWARD, A.; DIXON, L.; LEACH, S.; SMALL, R.; HITE, R. D.; HAPONIK, E.. Early intensive care unit mobility therapy in the treatment os acute respiratory failure. Crit Care Med. v.36, n.8, p.2238-22-43, 2008.

PEREIRA, M. C.. Mobilização precoce no paciente crítico. In: SANDRI, P.; GUIMARÃES, H. P.. Manual prático de fisioterapia no pronto socorro e UTI. 1 ed. São Paulo: Atheneu, 2014
SCHWEICKERT, E. D.; POHLMAN, M. C.; POHLMAN, A. S.; NIGOS, C.; PAWLIK, A. J.; ESBROOK, C. L.; SPEARS, L.; MILLER, M.; FRANCZYK, M.; DEPRIZIO, D.; SCHMIDT, G. A.; BOWMAN, A.; BARR, R.; MCCALLISTER, K. E.; HALL, J. B.; KRESS, J. P.. Early physical and occupational therapy in mechanically ventilated, critically ill patients: a randomized controlled trial. Lancet, p.1874-1828, 2009. DOI:

https://10.1016/S0140-6736(09)60658-9

SILVA, V. S.; PINTO, J. G.; MARTINEZ, B. P.; CAMELIER, F. W. R.. Mobilização na unidade de terapia intensiva: revisão sistemática. Revista Fisioter Pesq., v.21, n.4, p.394-404, 2014.

A CBPC - Companhia Brasileira de Produção Científica (CNPJ: 11.221.422/0001-03) detém os direitos materiais desta publicação. Os direitos referem-se à publicação do trabalho em qualquer parte do mundo, incluindo os direitos às renovações, expansões e disseminações da contribuição, bem como outros direitos subsidiários. Todos os trabalhos publicados eletronicamente poderão posteriormente ser publicados em coletâneas impressas sob coordenação da Sustenere Publishing, da Companhia Brasileira de Produção Científica e seus parceiros autorizados. Os (as) autores (as) preservam os direitos autorais, mas não têm permissão para a publicação da contribuição em outro meio, impresso ou digital, em português ou em tradução. 